\title{
Impact of Random Telegraph Noise Profiles on Drain-Current Fluctuation during Dynamic Gate Bias
}

\author{
W. Feng, Member, IEEE, C.M. Dou, non-Member, M. Niwa, IEEE Fellow, IEEE, K. Yamada, \\ non-Member, and K. Ohmori, Member, IEEE
}

\begin{abstract}
The influence of random telegraph noise (RTN) in MOSFETs on drain current $\left(I_{d}\right)$ during the rise/fall edges of the pulsed gate voltage $\left(V_{g}\right)$ cycle was investigated. We have revealed for the first time that the existence of RTN increases $I_{d}$ fluctuations under dynamic $V_{\mathrm{g}}$ by making a comparison between FETs with and without RTN. The initial trap occupation states before varying $V_{g}$, which are governed by the RTN profiles, significantly affect the $I_{d}$ values during the rise/fall edges of $V_{g}$. The revealed effects of RTN with different profiles on $I_{d}$ under dynamic $V_{g}$ will be useful for designing ultra-high speed circuits.
\end{abstract}

Index Terms - MOSFETs, Random Telegraph Noise, Dynamic Gate Bias

\section{INTRODUCTION}

A $S$ metal-oxide-semiconductor (MOS) field-effect transistors (FETs) are scaled down for further integration, the relative impact of randomly placed discrete charges (oxide traps, interface states, and fixed charges) on device characteristics increases significantly, leading to circuit failure. One of the principal issues that has been studied is that of random telegraph noise (RTN) because of its increasing influence with shrinkage of the device area. ${ }^{1-4}$ Recently, RTN has attracted much attention in digital circuits, since it causes threshold voltage $\left(\mathrm{V}_{\text {th }}\right)$ variation and directly degrades circuit reliability. ${ }^{4}$ For example, the operation of SRAM devices depends on the current balance between FETs, and thus is very sensitive to $\mathrm{V}_{\text {th }}$ shifts. ${ }^{5}$ The RTN-induced failure of SRAMs during accelerated testing was observed. ${ }^{6}$ The influence of RTN during continuous ac cycles and the relationship to hysteresis behavior are also reported. ${ }^{7}$

In the operation of ultrahigh-speed circuits, the rectangular shape of the ideal large-signal is rounded as the clock frequency increases to several $\mathrm{GHz}$, so that the relative proportion of the rise and fall edges to the 'on' state becomes significant. However, the effect of RTN during the rise and fall edges is not

Manuscript received October 31, 2013. This work was supported by JST-CREST. W. Feng, M. Niwa, K. Yamada, and K. Ohmori are with the Graduate School of Pure and Applied Sciences, University of Tsukuba, 1-1-1 Tennnodai, Tsukuba, Ibaraki 305-8573, Japan and Core Research for Evolutional Science and Technology, Japan Science and Technology Agency (JST-CREST), Chiyoda, Tokyo 102-0075, Japan (e-mail: wei.feng@aist.go.jp).

C.M. Dou is with Honors Graduate Program for Nanotech/Sciences, University of Tsukuba, 1-1-1 Tennnodai, Tsukuba, Ibaraki 305-8573, Japan, and Tokyo Institute of Technology, Yokohama 226-8502, Japan. fully understood yet. Therefore, it is important to investigate the impact of RTN on dynamic drain current $\left(\mathrm{I}_{\mathrm{d}}\right)$ fluctuations during the rise/fall edges of the pulsed gate voltage $\left(V_{\mathrm{g}}\right)$.

In this work, the impact of RTN on $I_{d}$ fluctuations under dynamic gate bias is presented by making comparisons between FETs w/o and with RTN of different profiles. We have revealed that the profiles of RTN determine the $I_{d}$ fluctuation during the rise and fall edges of pulsed $\mathrm{V}_{\mathrm{g}}$.

\section{EXPERIMENTAL PROCEDURE}

We used planar-type n-FETs with a poly-Si/high-k gate stack structure for the characterization of the impact of RTN on $I_{d}$ fluctuations. An $\mathrm{HfO}_{2}$ gate dielectric film with a 0.8-nm-thick $\mathrm{SiO}_{2}$ interfacial layer was adopted for the gate-stack structure. The equivalent oxide thickness of the samples was $1.2 \mathrm{~nm}$. The gate length and width are 140 and $200 \mathrm{~nm}$, respectively. Fast IV units (Agilent B1530A) were employed for monitoring the $I_{d}$ fluctuations. Either constant dc biases or dynamic $V_{\mathrm{g}}$ pulsed inputs were applied on the FETs, while the drain voltage was maintained at $1 \mathrm{~V}$. In order to obtain fast, accurate $I_{d}$ responses to the fast dynamic $V_{\mathrm{g}}$, a common ground was taken at the measuring probe tips for the source, the drain, the gate and the substrate of the FET. We have characterized the noise properties of 16 FETs, from which three representative FETs are shown, namely w/o RTN, with symmetric RTN, and with asymmetric RTN.

\section{DYNAMIC FLUCTUATION}

For FETs with RTN, $I_{d}$ exhibits high and low levels due to the emission and capture states of the traps, respectively. The time constants of RTN ( $\tau_{\mathrm{c}}$ and $\tau_{\mathrm{e}}$ ) are defined as the average duration times of the high and low $\mathrm{I}_{\mathrm{d}}$ states, respectively. The values of $\tau_{\mathrm{c}}$ and $\tau_{\mathrm{e}}$ are generally used to understand the characteristics of the traps, such as trap energy and trap depth. ${ }^{4}$ We focus on the symmetry of the RTN profiles, which is reflected in the ratio of capture-to-emission times $\left(\tau_{\mathrm{c}} / \tau_{\mathrm{e}}\right)$. The $\tau_{\mathrm{c}} / \tau_{\mathrm{e}}$ ratio is governed by the difference between the trap energy $E_{T}$ and the Fermi level $E_{F}$, as shown in the following equation ${ }^{8}$

$$
\frac{\tau_{c}}{\tau_{e}}=\exp \left(\frac{E_{T}-E_{F}}{k_{B} T}\right)
$$

where $k_{B}$ is the Boltzmann constant and $T$ is temperature. A trap with different $E_{T}-E_{F}$ exhibits varied symmetry.

We investigated $I_{d}$ fluctuations for FETs with RTN of 
different profiles. In these experiments, RTN is observed in the $\mathrm{V}_{\mathrm{g}}$ ranges from $\mathrm{V}_{\mathrm{th}}+0.1 \mathrm{~V}$ to $\mathrm{V}_{\mathrm{th}}+0.4 \mathrm{~V}$ for two devices, i.e., FET-A and FET-B. The $\mathrm{V}_{\text {th }}$ values for FET-A and -B are $0.77 \mathrm{~V}$ and $0.76 \mathrm{~V}$, respectively. For the same condition of $\mathrm{V}_{\mathrm{g}}=\mathrm{V}_{\text {th }}+$ $0.25 \mathrm{~V}$, the FET-A exhibits symmetric RTN (Fig. 1 (a)), while FET-B shows asymmetric RTN (Fig. 1 (b)). These symmetric and asymmetric characteristics of RTN are more clearly demonstrated in the $I_{d}$ histograms Fig. 1 (c) and (d), respectively. Two primary $I_{d}$ peaks are observed in both cases, corresponding to the capture and emission conditions of RTN. Figure 1 (c) shows two peaks with similar $\mathrm{I}_{d}$ counts for FET-A, reflecting a $\tau_{\mathrm{c}} / \tau_{\mathrm{e}}$ ratio of 0.84 . For FET-B, the $\tau_{\mathrm{c}} / \tau_{\mathrm{e}}$ ratio is evaluated to be 4.2 .

The symmetry of RTN, which is reflected in the $\tau_{c} / \tau_{e}$ ratio, depends on $V_{g}$ and plays a crucial role in determining $I_{d}$ fluctuations under dc bias. Taking FET-B as an example, the changes in the time constants $\left(\tau_{\mathrm{c}}\right.$ and $\tau_{\mathrm{e}}$ ) of RTN and the corresponding standard deviation of the $I_{d}\left(\sigma_{I d}\right)$ values as functions of $I_{d}$ and $V_{g}$ are shown in Fig. 2. The $\sigma_{\text {Id }}$ value is strongly affected by the symmetry of RTN, showing a maximum at $\tau_{\mathrm{c}} / \tau_{\mathrm{e}}=1 .{ }^{9}$ The $\sigma_{\mathrm{Id}}$ exhibits a maximum at $\mathrm{V}_{\mathrm{g}}-\mathrm{V}_{\text {th }}$ $=0.3 \mathrm{~V}$, where $\tau_{\mathrm{e}}$ is almost at the same level as $\tau_{\mathrm{c}}$.

$I_{d}$ fluctuations were investigated under dynamic $V_{g}$. The waveforms of $V_{g}$ and the corresponding $I_{d}$ for 400 cycles are shown in Fig. 3 (a). $V_{g}$ is programmed to rise from the lower gate voltage $\left(\mathrm{V}_{\mathrm{gL}}\right)$ to the higher gate voltage $\left(\mathrm{V}_{\mathrm{gH}}\right)$ with the transition time $\left(\mathrm{t}_{\mathrm{TR}}\right)$ ranging from $10 \mu \mathrm{s}$ to $10 \mathrm{~ms}$. $\mathrm{V}_{\mathrm{g}}$ remains at $\mathrm{V}_{\mathrm{gH}}$ for $10 \mu \mathrm{s}$, and then decreases back to $\mathrm{V}_{\mathrm{gL}}$ with the same $\mathrm{t}_{\mathrm{TR}}$. According to the condition for the appearance of RTN in FETs under constant dc bias, the values of $\mathrm{V}_{\mathrm{gL}}$ and $\mathrm{V}_{\mathrm{gH}}$ are set to be $\mathrm{V}_{\text {th }}+0.1 \mathrm{~V}$ and $\mathrm{V}_{\text {th }}+0.4 \mathrm{~V}$, respectively. In order to make a fair comparison between the dynamic $\mathrm{I}_{\mathrm{d}}$ fluctuations during the rise and fall edges, the $I_{d}$ fluctuation was extracted at the middle points $\mathrm{V}_{\mathrm{gM}}\left(=\mathrm{V}_{\mathrm{th}}+0.25 \mathrm{~V}\right)$ between $\mathrm{V}_{\mathrm{gL}}$ and $\mathrm{V}_{\mathrm{gH}}$ to ensure the same transition time from $\mathrm{V}_{\mathrm{gL}}$ and $\mathrm{V}_{\mathrm{gH}}$ to the evaluating points, as shown in the magnified figure (Fig. 3 (b)). Figure 3 (c) shows the extracted $I_{d}$ values for 400 cycles. The cycle is repeated 200-4400 times to enable sufficient sampling of $I_{d}$ for fluctuation analysis.

The influence of RTN on $I_{d}$ during the gate voltage ramping (rise/fall) was examined by comparing the $I_{d}$ fluctuations among the FETs w/o, with symmetric, and with asymmetric RTN. Figure 4 (a) shows the $\sigma_{\text {Id }}$ of the FETs as a function of $t_{T R}$. The $\sigma_{\text {Id }}$ levels under dc bias for the FETs with and w/o RTN are added as references. Note that the magnitude of the asymmetric RTN (FET-B) is larger than that of the symmetric RTN (FET-A) as shown in Fig. 1. Therefore, the $\sigma_{\text {Id }}$ level under dc bias of the FET with asymmetric RTN is higher than that of the FET with symmetric RTN in Fig. 4 (a). As expected from the previous discussion in Fig. 2 (b), the appearance of RTN increases $\sigma_{\mathrm{Id}}$. The possibility of two trap states for the FETs with RTN results in larger values of dynamic fluctuation $\sigma_{\text {Id }}$ than that without RTN, reflecting the magnitudes of deviation under dc bias.

The increases in $\sigma_{\mathrm{Id}}$ values with $\mathrm{t}_{\mathrm{TR}}$ for FET-A and -B were
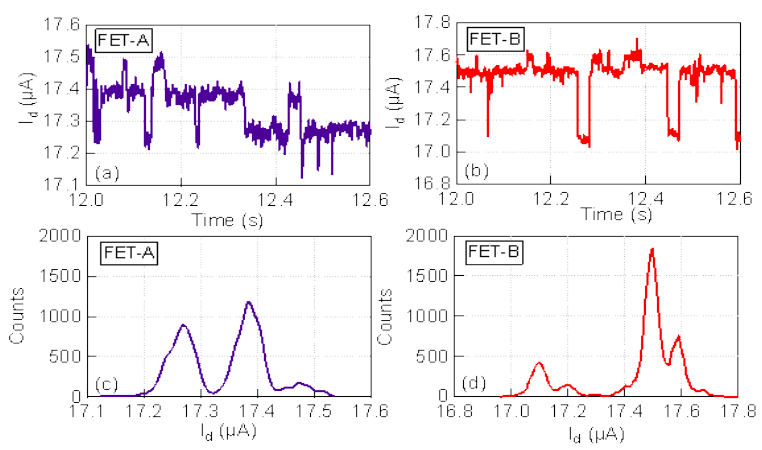

Fig. 1. (Color online) The $I_{d}$ fluctuation of (a) FET-A with symmetric RTN and (b) FET-B with asymmetric RTN measured under constant dc bias. $I_{d}$ distribution of (c) FET-A and (d) FET-B.
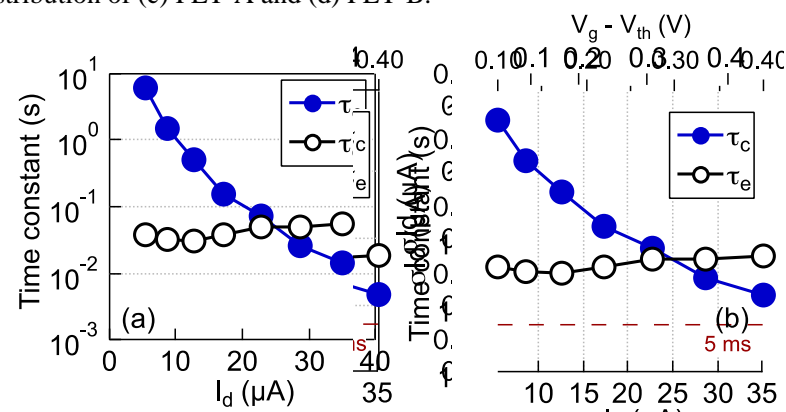

Fig. 2. (Color online) (a) Time constant of RTN and (b) $\sigma_{\text {Id }}^{\prime}$ of FET-B under dc bias as a function of $I_{d}$ and gate overdrive $V_{g}-V_{\text {th }}$. Note that the upper scales in (a) and (b) are not linear.
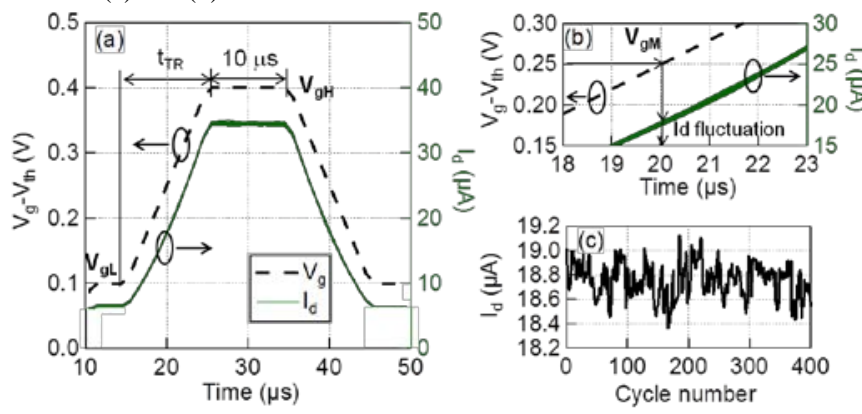

Fig. 3. (Color online) (a) $V_{g}$ pulse sequence and experimental data of $I_{d}$ as a function of time with $t_{\mathrm{TR}}=10 \mu \mathrm{s}$. (b) Magnified figure demonstrates the evaluation of the $I_{d}$ fluctuation of repeated $V_{g}$ pulses at $V_{g M}$. (c) Dynamic $I_{d}$ fluctuation values at $\mathrm{V}_{\mathrm{gm}}$ as a function of cycle number.
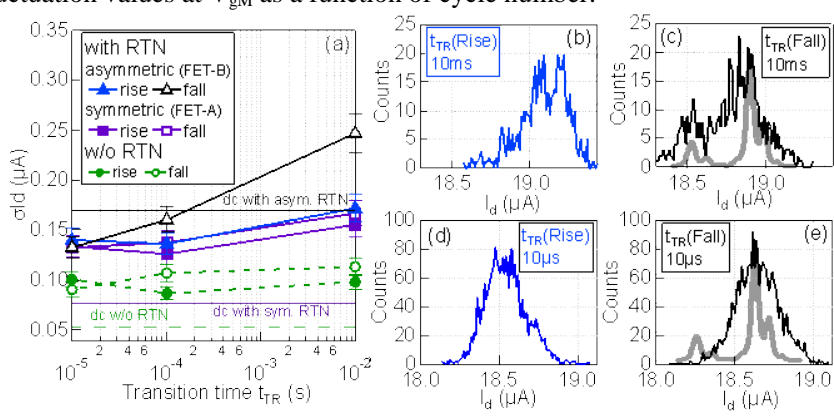

Fig. 4. (Color online) (a) Comparison of $\sigma_{\text {Id }}$ of the FET w/o RTN and with RTN of different profiles. Distribution of dynamic $I_{d}$ of the FET with asymmetric RTN in FET-B is shown for (b) rise and (c) fall edges with $\mathrm{t}_{\mathrm{TR}}=$ $10 \mathrm{~ms}$ and (d) rise and (e) fall edges of $\mathrm{t}_{\mathrm{TR}}=10 \mu \mathrm{s}$.

investigated in detail. The $I_{d}$ values of the FETs with RTN depend on the trap occupation states. Under dynamic $V_{g}$, there are two predominant factors for determining $I_{d}$ fluctuations: the initial trap occupation states and the trap occupation states change probabilities from initial $\mathrm{V}_{\mathrm{g}}$ to $\mathrm{V}_{\mathrm{gM}}$. The trap occupation 
states change due to the capture/emission of electrons, and the capture/emission probability depends on the ratio of the duration time $\left(\mathrm{t}_{\mathrm{TR}} / 2\right)$ to the time constants of RTN.

For FET-A, the values of $\tau_{\mathrm{c}} / \tau_{\mathrm{e}}$ under constant dc bias are 1.5, 0.84 , and 0.4 for $\mathrm{V}_{\mathrm{gL}}, \mathrm{V}_{\mathrm{gM}}$, and $\mathrm{V}_{\mathrm{gH}}$, respectively. As $\tau_{\mathrm{c}} / \tau_{\mathrm{e}}$ is closer to 1 at $\mathrm{V}_{\mathrm{gM}}$ than that at $\mathrm{V}_{\mathrm{gL}}$, according to previous discussion in Fig. 2, $\sigma_{\mathrm{Id}}$ under constant dc bias of $\mathrm{V}_{\mathrm{gM}}$ is larger than that of $\mathrm{V}_{\mathrm{gL}}$. When $\mathrm{V}_{\mathrm{g}}$ rises from $\mathrm{V}_{\mathrm{gL}}$ to $\mathrm{V}_{\mathrm{gM}}$, $\sigma_{\mathrm{Id}}$ changes towards to a larger value with the capture/emission of electrons. The capture/emission probability increases with $\mathrm{t}_{\mathrm{TR}}$. Therefore, $\sigma_{\mathrm{Id}}$ at $\mathrm{V}_{\mathrm{gM}}$ on the rise edges increases with $\mathrm{t}_{\mathrm{TR}}$. For the same reason, $\sigma_{\mathrm{Id}}$ at $\mathrm{V}_{\mathrm{gM}}$ of the fall edges also increases with $\mathrm{t}_{\mathrm{TR}}$.

For the FET-B, since $\tau_{\mathrm{c}} / \tau_{\mathrm{e}}$ is closer to 1 at $\mathrm{V}_{\mathrm{gm}}$ than that at $\mathrm{V}_{\mathrm{gL}}$ or $\mathrm{V}_{\mathrm{gH}}$ (Fig. 2(a)), a similar feature to that of FET-A is observed. However, as $\mathrm{t}_{\mathrm{TR}}$ becomes longer, the $\sigma_{\mathrm{Id}}$ value for the fall edges increases dramatically. To reveal the origin of the $\sigma_{\text {Id }}$ values, a histogram of dynamic $I_{d}$ for FET-B was evaluated for the rise and fall edges at $\mathrm{t}_{\mathrm{TR}}=10 \mathrm{~ms}$ and $10 \mu \mathrm{s}$ (Fig. 4 (b)-(e)). For the case of $\mathrm{t}_{\mathrm{TR}}=10 \mathrm{~ms}$, the $\mathrm{I}_{\mathrm{d}}$ distribution at the rise edges mainly shows one peak, while that for the fall edges is composed of two peaks. Therefore, $\sigma_{\text {Id }}$ of the fall edges shows larger values. The $I_{d}$ distribution under constant dc bias is superimposed to the $I_{d}$ histogram of the fall edges, as shown by the grey line with its horizontal scale shifted. We emphasize that the difference in peak positions of $I_{d}$ distribution under dynamic $\mathrm{V}_{\mathrm{g}}$ is similar to that of constant dc bias, indicating the appearance of RTN during the fall edge. Note that the $I_{d}$ distribution of dynamic $\mathrm{V}_{\mathrm{g}}$ is broader than that under dc bias, resulting from additional $I_{d}$ fluctuations due to the dynamic transition of $\mathrm{V}_{\mathrm{g}}$. With a shorter $\mathrm{t}_{\mathrm{TR}}$ of $10 \mu \mathrm{s}$, the $\mathrm{I}_{\mathrm{d}}$ distribution width becomes narrower than that of the $10 \mathrm{~ms}$ case. The $\mathrm{I}_{d}$ distribution of the rise edges remains a single peak-shaped structure (Fig. 4 (d)). However, the two peak-shaped structure of the $I_{d}$ distribution when $t_{T R}=10 \mathrm{~ms}$ on the fall edges becomes one peak-shaped at $10 \mu \mathrm{s}$, as shown in Fig. 4 (e).

Here we consider the origins of the one or two peaks in the $I_{d}$ distribution. As $\tau_{c}$ is the time for the empty states, the value of $\tau_{\mathrm{c}} / \tau_{\mathrm{e}}$ indicates the possibility of empty traps. With a $\tau_{\mathrm{c}} / \tau_{\mathrm{e}}$ value of 170 at $\mathrm{V}_{\mathrm{gL}}$, the possibility of empty traps is $99.4 \%$. For $\mathrm{t}_{\mathrm{TR}}=$ $10 \mathrm{~ms}$, the time for capturing electrons during the rise edges $\left(t_{T R} / 2\right)$ is less one order smaller than the $\tau_{c}$ values as shown in Fig. 2 (a). Therefore, the trap occupation state at $\mathrm{V}_{\mathrm{gM}}$ on the rise edges is mostly empty, resulting in a single peak-shaped structure for the $I_{d}$ distribution in Fig. 4 (b). The trapping of electrons changes the trap occupation states from empty to filled, which increases $\sigma_{\text {Id }}$. The electron capture probability is larger for longer $\mathrm{t}_{\mathrm{TR}}$, resulting in an increase in $\sigma_{\mathrm{Id}}$.

In the case of the fall edges, the value of $\tau_{\mathrm{c}} / \tau_{\mathrm{e}}$ at $\mathrm{V}_{\mathrm{gH}}$ decreases to 0.26 , indicating the possible existence of both empty and filled states. Thus, there are two kinds of initial trap occupation states for the fall edges, which result in a histogram with two peaks (Fig. 4 (c)). For a shorter rising time $\mathrm{t}_{\mathrm{TR}}$ of $10 \mu \mathrm{s}$, which is three orders smaller than the $\tau_{\mathrm{c}}$ value in Fig. 2 (a), the capture probability decreases to the order of $1 / 1000$. Therefore, the trap occupation states remain the same as that at $\mathrm{V}_{\mathrm{gL}}$ (empty) with a high probability for $\mathrm{V}_{\mathrm{gH}}$. Hence, one initial trap occupation state at $\mathrm{V}_{\mathrm{gH}}$ results in a single peak-shaped structure for the $I_{d}$ distribution at the fall edges (Fig. 4 (e)).

Two kinds of initial trap occupancy states for the repeated $V_{g}$ pulses lead to a two peak-shaped structure for the dynamic $I_{d}$ distribution, resulting in a larger $\sigma_{\text {Id }}$ than the case of the single initial trap state. Therefore, the variation of the initial occupancy trap states is essential for the $I_{d}$ fluctuation during the rise and fall edges of dynamic $\mathrm{V}_{\mathrm{g}}$.

\section{CONCLUSION}

We have investigated the influence of RTN during dynamic pulses upon $I_{d}$ fluctuation, focusing on the rise and fall edges. We have found for the first time that RTN increases dynamic $I_{d}$ fluctuations, even when the transition time is significantly shorter than the time constants $\left(\tau_{\mathrm{c}}\right.$ and $\tau_{\mathrm{e}}$ ) of RTN. The initial trap occupation states prior to the rise/fall edges of $\mathrm{V}_{\mathrm{g}}$ are reflected in the $I_{d}$ distribution during rise/fall ramping. The initial trap occupation states are governed by the symmetry of the RTN profiles $\left(\tau_{\mathrm{c}} / \tau_{\mathrm{e}}\right)$ in addition to the magnitudes of $\tau_{\mathrm{c}}$ and $\tau_{\mathrm{e}}$. Therefore, the profiles of RTN are essential for determining the $\mathrm{I}_{\mathrm{d}}$ fluctuation during the dynamic $\mathrm{V}_{\mathrm{g}}$.

These results imply an additional factor in the $I_{d}$ noise modeling of FETs for designing an ultra-high speed circuits, where the proportion of the rise/fall time to a single large-signal clock is not negligible.

\section{ACKNOWLEDGMENT}

The authors would like to thank Innovative Center Advanced Nanodevices (ICAN) of Advanced Industrial Science and Technology (AIST) for the continuous support.

\section{REFERENCES}

[1] R. E. Howard, L. D. Jackel, P. M. Mankiewich, and W. J. Skocpol, "Electrons in silicon microstructures," Science, vol. 231, no. 4736, pp. 346-349, Jan. 1986.

[2] K. Ohmori, W. Feng, S. Sato, R. Hettiarachchi, M. Sato, T. Matsuki, K. Kakushima, H. Iwai, and K. Yamada, "Direct real-time observation of channel potential fluctuation correlated to random telegraph noise of drain current using nanowire MOSFETs with four-probe terminals," in Proc. 2011 Symp. VLSI Technology, 2011, pp.202-203.

[3] K. Takeuchi, "Impact of discrete-charge-induced variability on scaled MOS devices,” IEICE Trans. Electron., vol. E95-C, no. 4, pp. 414-420, Apr. 2012.

[4] S. T. Martin, G. P. Li, E. Worley, and J. White, "The gate bias and geometry dependence of random telegraph signal amplitudes,” Electron Device Letters, vol. 18, no. 9, pp.444-446, Sep. 1997.

[5] S. O. Toh, T.-J. King Liu, and B. Nikolić, "Impact of random telegraph signaling noise on SRAM stability,” in Proc. Symp. VLSI Technology, 2011, pp. 204-205.

[6] K. Takeuchi, T. Nagumo, K. Takeda, S. Asayama, S. Yokogawa, K. Imai, and Y. Hayashi, "Direct observation of RTN-induced SRAM failure by accelerated testing and its application to product reliability assessment," in Proc. Symp. VLSI Technology, 2010, pp. 189-190.

[7] H. Miki, N. Tega, Z. Ren, C. P. D’Emic, Y. Zhu, D. J. Frank, M. A. Guillorn, D. Park, W. Haensch, and K. Torii, "Hysteretic drain-current behavior due to random telegraph noise in scaled-down FETs with high-k/metal-gate stacks,” in Proc. IEDM, 2010, pp. 620-623.

[8] M. J. Kirton, and M. J. Uren, "Noise in solid-state microstructures: A new perspective on individual defects, interface states and low-frequency $(1 / f)$ noise,” Advances in Physics, vol. 38, no.4, pp. 367-468, May. 1989.

[9] N. Zanolla, D. Šiprak, M. Tiebout, P. Baumgartner, E. Sangiorgi, and C. Fiegna, "Reduction of RTS noise in small-Area MOSFETs under switched bias conditions and forward substrate bias," Trans. on Electron Devices, vol. 57, no. 5, pp. 1119-1128, May 2010. 